\title{
Generalized Rest Mass and Dirac's Monopole in 5D Theory and Cosmology
}

Boris G. Aliyev ${ }^{\dagger}$

check for

updates

Citation: Aliyev, B.G. Generalized Rest Mass and Dirac's Monopole in 5D Theory and Cosmology. Universe 2021, 7, 295. http://doi.org/10.3390 /universe7080295

Received: 18 June 2021

Accepted: 4 August 2021

Published: 11 August 2021

Publisher's Note: MDPI stays neutral with regard to jurisdictional claims in published maps and institutional affiliations.

Copyright: (C) 2021 by the author. Licensee MDPI, Basel, Switzerland. This article is an open access article distributed under the terms and conditions of the Creative Commons Attribution (CC BY) license (https:// creativecommons.org/licenses/by/ $4.0 /)$.
Architecture Department, V.I. Surickov Moscow State Art Institute, Altendorfer Str. 28, 09113 Chemnitz, Germany; bgaliyev@mail.ru; Tel.: +49-371332-417-30

+ Retired.

\begin{abstract}
It is shown that the 5D geodetic equations and 5D Ricci identities give us a way to create a new viewpoint on some problems of modern physics, astrophysics, and cosmology. Specifically, the application of the 5D geodetic equations in $(4+1)$ and $(3+1+1)$ splintered forms obtained with the help of the monad and dyad methods made it possible to introduce a new, effective generalized concept of the rest mass of the elementary particle. The latter leads one to novel connections between the general relativity and quantum field theories, and all that, including the (4+1) splitting of the 5D Ricci identities, brings about a better understanding of the magnetic monopole problem and the vital difference in the origins of the Maxwell equations and gives rise to surprising connections between them. The obtained results also provide new insight into the mechanism of the 4D universe's expansion and its following acceleration.
\end{abstract}

Keywords: monad and dyad methods; effective generalized rest mass concept; scalar gravitational field; 5D geodetic equations; cylindrical symmetry (cylindricity) conditions; 5D Ricci identities; Maxwell equations; magnetic monopole; topological second-order phase transition in cosmology

\section{Introduction}

The article considers some old and new problems of modern physics, astrophysics, and cosmology in the framework of the 5D theory, using the well-developed and well-known for advanced researchers monad and dyad methods in general relativity and 5D theory (See [1], pp. 184-207 and [2-4] for more details). Reviewing the papers of the different old and modern authors who have worked in the framework of the 5D theories [5-9], one can see that almost all of them, as it was rigorously proved by V.A. Fock [5] and Yu.B. Rumer [8], came to the $5 \mathrm{D}$ optics under the standard requirement that ratio $e / m_{0}$ has to be constant. In this article, we show that cancelation of this requirement leads to new, fundamental and in some sense unexpected consequences, concerning the generalized effective rest mass concept $\left(m_{0} \rightarrow \hat{m}_{0}\right)$, and to the new ratio requirement $\left(e / \hat{m}_{0} \neq\right.$ const $)$. We reveal the physical grounds for the vital difference in the origins of the first and second pairs of the Maxwell equations and obtain unexpected functional connections between them. We also inspect the role of the 5D Ricci identities in the understanding of some of these problems. Moreover, we hope that our findings can improve the present understanding of the magnetic monopole's problem, as well as the other important problems of modern physics, astrophysics, and cosmology, including dark matter and dark energy. The results obtained make it possible to single out two ages in the universe's evolution, namely the ages of the magnetic monopoles and the electrons.

\section{The Basic Elements of the Monad and Dyad Methods in 5D Theory}

To begin with, I would like to recall that one of the first researchers to develop and then successfully apply the full monad method together with the cylindrical symmetry (cylindricity) condition in the 5D theory was Prof. Peter Gabriel Bergmann [10], with whom-and it was a great honor for me-I had a long-lasting friendship and collaboration. 
Here I want to briefly present the basic concepts of the monad method in 5D theory with the monad vector's chronometric gauging and the dyad method with the mixed gauging of the dyad vectors: first the chronometric and then the kinemetric or vice versa. These methods allow us to employ the procedure of the $V_{5}$ reductions: $V_{5} \mapsto V_{4} \mapsto V_{3}$ more correctly or, in other words, the orthogonal (4+1) splitting and then also the orthogonal $(3+1+1)$ splitting of the $V_{5}$ with the subsequent expression of the $5 \mathrm{D}$ theory in terms of the $4 \mathrm{D}$ and 3D physical-geometrical quantities. In the framework of the monad method with the chronometric gauging of the monad vectors we can represent the $5 \mathrm{D}$ metric $G_{A B}$ as follows: $G_{A B}=g_{A B}-\lambda_{A} \cdot \lambda_{B}$. Here we have chosen the signature of the $V_{5}$ to be equal $(+----)$. Later we will be convinced that this choice is a more preferable one. The indexes $A, B, C, \ldots=0,1,2,3,5$. The square of the $5 \mathrm{D}$ interval takes on the form $d I^{2}=G_{A B} \cdot d x^{A} \cdot d x^{B}=d s^{2}-d \lambda^{2}$. Here $d s^{2}=g_{A B} \cdot d x^{A} \cdot d x^{B}$ and $d \lambda=\lambda_{A} \cdot d x^{A}$. The space-like monad vectors $\lambda_{A}$ in chronometric gauging can be written as follows:

$$
\lambda_{A}=\frac{G_{A 5}}{\sqrt{-G_{55}}}, \lambda^{A}=\frac{G_{5}^{A}}{\sqrt{-G_{55}}}, \sqrt{-G_{55}} \equiv \varphi .
$$

The $\lambda_{A}$ is a tangential vector to the $x^{5}$ coordinate line and the orthogonal one to the space-time hypersurface $V_{4}$. Also $g_{A B}$ is the metric tensor of $V_{4}$. Then, the orthonormality condition gives us: $\lambda^{A} \cdot \lambda_{A}=-1 ; \lambda^{A} \cdot g_{A B}=0$; and of course, we have the well-known relations: $G^{A B} \cdot G_{A B}=5 ; G^{A B} \cdot G_{B C}=\delta_{C}^{A} ; g^{A B} \cdot g_{A B}=4 ; g^{A B} \cdot g_{B C}=\delta_{C}^{A}$. It should be also noted that $g_{5 A} \equiv 0$. Now we can construct the monad differentiation operators: $\lambda^{A} \cdot \partial_{A} \equiv \bar{\partial}_{\Lambda}^{+}$and $g_{\alpha}^{A} \cdot \partial_{A} \equiv \bar{\partial}_{\alpha}^{+}$. Then, with help of the latter ones we can build the $x^{5}$ and $V_{4}$ projected directional normal and covariant derivatives: $\bar{\nabla}_{\Lambda}^{+} \equiv \bar{\partial}_{\Lambda}^{+}$and $\bar{\nabla}_{\alpha}^{+}$, which have the standard form, being expressed through the operators $\bar{\partial}_{\alpha}^{+}$. In the case of the dyad method the $5 \mathrm{D}$ metric $G_{A B}$ and the square of the $5 \mathrm{D}$ interval $d I$ takes on the forms as it follows then here: $G_{A B}=\tau_{A} \cdot \tau_{B}-\lambda_{A} \cdot \lambda_{B}-h_{A B} ; d I^{2}=d \tau^{2}-d \lambda^{2}-d l^{2} ; d \tau=\tau_{A} \cdot d x^{A}$; $d l^{2}=h_{A B} \cdot d x^{A} \cdot d x^{B}$. Now we can also write down the orthonormal time-like vectors $\tau_{A}$ of the dyad in the kinemetric gauging as it follows below (the case of the space-like vectors $\lambda_{A}$ of the dyad was commented above):

$$
\tau^{A}=\frac{G^{0 A}}{\sqrt{G^{00}}}, \tau_{A}=\frac{G_{A}^{0}}{\sqrt{G^{00}}}, \tau^{A} \cdot \tau_{A}=1, \lambda^{A} \cdot \tau_{A}=0,
$$

and $\tau^{A} \cdot h_{A B}=\lambda^{A} \cdot h_{A B}=0$. Note that the 3D metric of the $V_{3}$ has the following wellknown properties: $h^{A B} \cdot h_{A B}=3, h^{A B} \cdot h_{B C}=-\delta_{C}^{A}$. It should be also noted that $h^{0 A}=$ $h_{5 A} \equiv 0$. Vector $\tau^{A}$ is the tangential one to the $x^{0}$ coordinate line. We can also build the same type of differentiation dyad operators: $\bar{\partial}_{\tau}^{+}, \bar{\partial}_{\Lambda}^{+}$are the $\tau^{A}$ and $\lambda_{A}$ projected directional normal derivatives, and $-h_{i}^{A} \cdot \partial_{A} \equiv \bar{\partial}_{i}^{+} \rightarrow \bar{\nabla}_{i}^{+}$. Here $\bar{\nabla}_{i}^{+}$stands for the $V_{3}$ projected covariant derivative of the standard above-mentioned form. The operator $\bar{\partial}_{\tau}^{+}$is here defined as follows below:

$$
\bar{\partial}_{\tau}^{+} \bar{B}_{k \ldots}^{i \ldots}=\tau^{A} \cdot \partial_{A} \bar{B}_{k \ldots}^{i \ldots}+\bar{N}_{k}^{l} \cdot \bar{B}_{l \ldots}^{i \ldots}+\ldots-\bar{N}_{l}^{i} \cdot \bar{B}_{k \ldots}^{l \ldots}-\ldots
$$

Here indexes $i, j, k, \ldots=1,2,3$, and $\tau^{A}$ connectivity (an analogy of the Christoffel symbols for the operator $\bar{\partial}_{\tau}^{+}$): $\bar{N}_{i}^{k}=\tau^{A} \cdot \bar{\partial}_{i}^{+} h_{A}^{k}$. We will mark these projected quantities with a tilde over the operators. The $(4+1)$ splitting of the 5D relations one can produce then with the help of the monad method by making the inner product of the ones with the metric elements as $\lambda_{A}, g_{A B}$, and its combinations. The same $(3+1+1)$ splitting of the 5D relations one can produce with the help of the dyad method by making the inner product of the ones with the metric elements as $\tau_{A}, \lambda_{A}, h_{A B}$, and its combinations. See [1-3], examples below, and also the Appendix A for more details. 


\section{The New Rest Mass Concept and Some Cosmological Problems}

Let us consider the geodetic equation in 5D theory, which has the following standard form: $G^{A} \equiv \frac{D^{2} x^{A}}{d I^{2}}=0$. Evidently, one can obtain it if one will use the 5D action $\Sigma=-m_{0} \cdot c \cdot \int d I$ as it follows below (here $m_{0}$ is an initial rest mass of the $5 \mathrm{D}$ test particle and $c$ is the light velocity):

$$
G^{A} \equiv \frac{d^{2} x^{A}}{d I^{2}}+P_{B C}^{A} \cdot \frac{d x^{B}}{d I} \cdot \frac{d x^{C}}{d I}=0
$$

Here the square of the 5D interval $d I^{2}=G_{A B} \cdot d x^{A} \cdot d x^{B}$ and the indexes $A, B, C, \ldots=$ $0,1,2,3,5 . G_{A B}$ is the 5D metric of the $V_{5}$ and $P_{B C}^{A}$ stands for the 5D Christoffel symbols. It should be added that these values of the indexes are connected with such a fact that we consider the 5D Kaluza and Klein theories separately and we believe that 5D Kaluza theory is connected with the electromagnetism (the charge $Q_{0}$ of the particle, index 5) and 5D Klein theory is connected with the rest mass $m_{0}$ of the particles (index 4). Thus, actually we have a variant of the $6 \mathrm{D}$ theory, but this variant is not developed yet. Making here the $(4+1)$ splitting with the help of the monad method (see the examples below) and eliminating the 5th coordinate, then imposing the cylindrical symmetry (cylindricity) condition along the 5th coordinate (it means that we have the Killing equations for the $5 \mathrm{D}$ metric $G_{A B}$ along the tangent vector to the fifth coordinate $\xi_{A}=\varphi \cdot \lambda_{A}$ : the Lie derivatives $L_{\xi} G_{A B}=\xi_{A ; B}+\xi_{B ; A}=0$ ) one can obtain from Equation (4) the following system:

$$
\begin{gathered}
m_{0} \cdot \lambda_{A} \cdot G^{A}=\frac{D^{+}}{d s}(\varphi \cdot \hat{p})=0 \Rightarrow \varphi \cdot \hat{p}=\text { const. }=\frac{n \cdot e}{2 \cdot \sqrt{k_{0}}}=n \cdot \hat{m}_{P l} ; \\
m_{0} \cdot g_{A}^{\alpha} \cdot G^{A}=0 \Rightarrow \frac{D^{+\hat{p}^{\alpha}}}{d s}=\frac{2 \cdot \sqrt{k_{0}}}{c^{2}} \cdot \phi \cdot \hat{p} \cdot \bar{u}^{\beta} \cdot F_{\beta}^{\alpha}+\bar{\partial}^{\alpha+} \hat{m}_{0}
\end{gathered}
$$

Then, inserting the integral of Equations (5) and (6) and choosing this integral to be corresponding to the 4D Lorentz force (one can easily understand that only in this case the first term in the r.h.s. of Equation (6) becomes equal to the first term in the r.h.s. of Equation (7)) one can easily obtain the $4 \mathrm{D}$ geodetic equation in 5D theory as it follows below (see Appendix A for more details):

$$
m_{0} \cdot g_{A}^{\alpha} \cdot G^{A}=0 \Rightarrow \frac{D^{+\hat{p}^{\alpha}}}{d s}=\frac{Q_{0}}{\hat{m}_{0} \cdot c^{2}} \cdot \hat{\bar{p}}^{\beta} \cdot F_{\beta}^{\alpha}+\bar{\partial}^{\alpha+} \hat{m}_{0} .
$$

Here $k_{0}$ is the Newtonian gravitational constant, $Q_{0}=n \cdot e$ ( $e$ is the electric charge of an electron), and also we have put here the hypothetical 5D "Plank mass" $\hat{m}_{P l}=$ $e /\left(2 \cdot \sqrt{k_{0}}\right) \approx 10^{-6} g$. It should be also added that here we have $\frac{D^{+}}{d s}=\bar{u}^{\alpha} \cdot \bar{\nabla}_{\alpha}^{+}-\hat{u} \cdot \bar{\partial}_{\Lambda}^{+}, F_{\alpha \beta}=$ $\bar{\nabla}_{\alpha}^{+} A_{\beta}-\bar{\nabla}_{\beta}^{+} A_{\alpha}$ is a tensor of the electromagnetic field, and $A_{\alpha}$ is a $4 \mathrm{D}$ vector-potential of this field, $\hat{u}=d \lambda / d s$ is a "velocity" along the fifth coordinate, and the $4 \mathrm{D}$ velocity $\bar{u}^{\alpha}=d \bar{x}^{\alpha} / d s$. Here the indexes $\alpha, \beta, \gamma, \ldots=0,1,2,3$. It should be noted that by using here the monad method we have introduced by a natural way the effective generalized rest mass of the Lorentz type $\hat{m}_{0}$ in the following single-valued, unique form [2-4,11]. We have again: $d I^{2}=d s^{2}-d \lambda^{2} ; \hat{m}_{0}=m_{0} \cdot \hat{\beta} ; \hat{p}=\hat{m}_{0} \cdot \hat{u} ; \hat{p}^{\alpha}=\hat{m}_{0} \cdot \bar{u}^{\alpha}$ and $\hat{\beta}=\left(1-\hat{u}^{2}\right)^{-1 / 2}$ is a $5 \mathrm{D}$ Lorentz factor.

It should be also noted that Equations (5)-(7) for the 5D test particle one can also obtain from the above-mentioned $5 \mathrm{D}$ action if vary it, due to the cylindricity condition, independently on $x^{\alpha}$ and $x^{5}$ [4]:

$$
\Sigma=-m_{0} \cdot c \cdot \int d I=-c \cdot \int \hat{m}_{0} \cdot d s+\frac{2 \cdot \sqrt{k_{0}}}{c} \cdot \int \varphi \cdot \hat{p} \cdot A_{\alpha} \cdot d \bar{x}^{\alpha}-c \cdot \int \varphi \cdot \hat{p} \cdot d x^{5} .
$$


Here one can easily obtain the standard action for the test charged particle up to the gage invariant term, inserting an integral of Equation (5):

$$
\Sigma=-c \cdot \int \hat{m}_{0} \cdot d s+\frac{Q_{0}}{c} \cdot \int A_{\alpha} \cdot d \bar{x}^{\alpha}-n \cdot \hat{m}_{P l} \cdot c \cdot x^{5}=S_{\hat{m}_{0}}+S_{e}-n \cdot \hat{m}_{P l} \cdot c \cdot x^{5} .
$$

Now let us consider the 5D Hamilton-Jacoby equation for the charged test particle:

$$
G^{A B} \cdot \partial_{A} \Sigma \cdot \partial_{B} \Sigma-m_{0}^{2} \cdot c^{2}=0 .
$$

After (4+1) splitting this equation will take the following form:

$$
g^{\alpha \beta} \cdot\left(\bar{\partial}_{\alpha}^{+} \hat{S}-\frac{Q_{0}}{c} \cdot A_{\alpha}\right) \cdot\left(\bar{\partial}_{\beta}^{+} \hat{S}-\frac{Q_{0}}{c} \cdot A_{\beta}\right)-\hat{m}_{0}^{2} \cdot c^{2}=0
$$

where we have put: $\hat{S} \equiv S_{\hat{m}_{0}}$.

Here one can be convinced again that in the 5D theory one can always obtain the effective generalized rest mass $\hat{m}_{0}$ if one performs $(4+1)$ splitting. One more confirmation of this transition principle in the 5D theory one can obtain when one considers the transition to the quantum theory. In this case, as it is well-known, the 5D equation for the wave function $\Psi$ has the following Klein-Gordon-Fock form:

$$
G^{A B} \cdot \nabla_{A} \nabla_{B} \Psi+\frac{m_{0}^{2} \cdot c^{2}}{\hbar^{2}} \cdot \Psi=0
$$

It should be noted here that this equation should have the Hamilton-Jacoby Equations (10) and (11) as the characteristic ones.

In the approximation of $5 \mathrm{D}$ optics one has the following relation for the wave function $\Psi$ :

$$
\Psi=C \cdot \psi \cdot \exp \left\{\frac{i}{\hbar} \cdot \frac{Q_{0} \cdot c}{2 \cdot \sqrt{k_{0}}} \cdot x^{5}\right\} .
$$

Here function $\psi$ does not depend on the $x^{5}$ coordinate. Then, one can make the $(4+1)$ splitting again and as a result, one can obtain the equations for the $5 \mathrm{D}$ or $4 \mathrm{D}$ wave functions $\Psi$ or $\psi$ as it follows below:

$$
\begin{gathered}
g^{\alpha \beta} \cdot \bar{\nabla}_{\alpha}^{+} \bar{\nabla}_{\beta}^{+} \Psi+\Phi^{\alpha} \cdot \bar{\nabla}_{\alpha}^{+} \Psi+\frac{\hat{m}_{0}^{2} \cdot c^{2}}{\hbar^{2}} \cdot \Psi=0 ; \\
\left(\bar{\nabla}^{+\alpha}+\frac{i \cdot Q_{0}}{\hbar \cdot c} \cdot A^{\alpha}+\Phi^{\alpha}\right) \cdot\left(\bar{\nabla}_{\alpha}+\frac{i \cdot Q_{0}}{\hbar \cdot c} \cdot A_{\alpha}\right) \cdot \psi+\frac{\hat{m}_{0}^{2} \cdot c^{2}}{\hbar^{2}} \cdot \psi=0
\end{gathered}
$$

The transition principle for the effective generalized rest mass is valid also in this case: $V_{5} \rightarrow V_{4} \Rightarrow m_{0} \rightarrow \hat{m}_{0}$. It should also be added that Louis de Broglie was the first to approach a close understanding of this transition principle of nature in the 5D theory (See [7] for more details). Unfortunately, he chose the wrong signature $(+---+)$ of the $V_{5}$ instead of the right one $(+----)$, as well as the restrictive requirement for the relation $e / m_{0}$ to be constant. Because of that he failed to establish the transition principle in its complete form and could not obtain the exact formula (18) for $\hat{m}_{0}$ (See below). Moreover, he has tried to develop the 5D theory together with this requirement beyond the 5D optics, though it was shown, as we have mentioned above, by V. A. Fock [5] and Yu.B. Rumer [8] that this requirement $\left(e / m_{0}=\right.$ const.) leads one only to the 5D optics. Because of this, he came to the contradiction and, as a result, he obtained an incomplete formula for the effective generalized rest mass that is valid in a certain special case solely. However, it is important to note that it was de Broglie who, considering the 5D wave equation and its connection with the quantum theory, later correctly understood that in the case of a 
charged test particle in 5D wave Equation (12) a certain invariant $I$ should be employed instead of the usual rest mass $m_{0}$. This invariant (Equation (41) in Ref. [7]):

$$
I=\sqrt{m_{0}^{2}+\frac{e^{2}}{16 \cdot \pi \cdot k_{0}}}
$$

is just a special case of the effective generalized rest mass $\hat{m}_{0}$ introduced in the present paper, see Equation (18) below. The latter is reduced to $I$ at $n=1$ and $\varphi=$ const $=$ $2 \cdot \sqrt{\pi}: \hat{m}_{0}=\omega(n ; \varphi) \Rightarrow I=\omega(1 ; 2 \cdot \sqrt{\pi})$. Thus, the effective generalized rest mass $\hat{m}_{0}$ could be considered as a generalization of de Broglie's hypothesis. It's very important that in his article he also made a very interesting and deep prophesy: «Si l'on parvient à interpréter la façon dont interviennent, dans l'équation de'onde a cinq dimensions, les constantes $e, m_{0}, c, \hbar$ et $k_{0}$, on sera bien près d'avoir compris quelques-uns des secrets les plus troublants de la Nature» (in French, see below an English translation) ${ }^{1}$. One can easily be convinced that only the introduction of the effective generalized mass $\hat{m}_{0}$ leads one to the compact and complete form of the geodetic Equation (7) and fulfills it with a large and important physical sense. When P.A.M. Dirac visited the Lomonosov Moscow State University (MSU), he took a piece of chalk, and wrote on the wall of the chair of theoretical physics' office: "The physical law should have mathematical beauty." It is easy to see that the geodetic Equation (7) has this mathematical beauty.

It is worth mentioning that the first time the author obtained Equations (5)-(7) and relation (18) was in 1978 and these results were reported at the seminar of my scientific adviser Prof. Yu.S. Vladimirov in the Lomonosov MSU in Moscow. Next, in 1979, they were reported and published in Russian in Minsk [11] and one year later, in 1980, in English in Germany (Jena, GDR) [2]. Unfortunately, the author found an article [7] only a few years later, about 1982, and it's necessary to stress that this great article of the outstanding physicist was not appreciated with dignity till now. It should be also noted here that only many years later, in 2015, T.X. Zhang in his very interesting article [9] found the correct expression for the so-called certain scale factor $f$, but unfortunately he did not connect this factor with the particle's mass (this connection follows from the results of the present paper and reads: $\left.\hat{m}_{0}=m_{0} \cdot f\right)$. Therefore, the $(4+1)$ splintered geodetic equations in his article [9] are very cumbersome and also contain several terms which do not have any clear physical meaning. Moreover, their form is also very far from the clear and compact form of Equation (7) (See [9], Equations (77) and (78) for comparison).

Probably, T.X. Zang was not familiar either with L.de Broglie's article [7] or with my articles [2,11,12], since in his paper [9] he claimed that he was the first who obtained the factor $f$ in the $5 \mathrm{D}$ theory. This is not the case. The first, in some sense, was L. de Broglie. Then, these results were independently obtained in Refs. [2-4,11,12]. Thus, to the best of my knowledge, T.X. Zhang was only the third in this row. In any case, we need to note that it is noteworthy that three independent researchers at different times and using different ways came to very close results. It permits one to believe earnestly that this new concept of the rest mass $\hat{m}_{0}$ is highly likely correct and makes our scientific horizons wider.

Then, let us briefly demonstrate below, as an example, the (4+1) splitting of the above-mentioned $5 \mathrm{D}$.

Take derivatives of the $5 \mathrm{D}$ metric $G_{A B}$. We will start with these Killing equations (See Appendix A below for more details and also examples):

$$
\begin{gathered}
\lambda^{A} \cdot \lambda^{B} \cdot L_{\xi} G_{A B}=\lambda^{A} \cdot \lambda^{B} \cdot\left(\xi_{A ; B}+\xi_{B ; A}\right)=-2 \cdot \bar{\partial}_{\Lambda}^{+} \varphi=0 \Rightarrow \bar{\partial}_{\Lambda}^{+} \varphi=0 ; \\
\lambda^{A} \cdot g_{\alpha}^{B} \cdot\left(\xi_{A ; B}+\xi_{B ; A}\right)=\varphi^{2} \cdot \bar{\partial}_{\Lambda}^{+}\left(\lambda_{\alpha} / \varphi\right)=\frac{2}{c^{2}} \sqrt{k_{0}} \cdot \varphi^{2} \cdot \bar{\partial}_{\Lambda}^{+} A_{\alpha}=0 \Rightarrow \bar{\partial}_{\Lambda}^{+} A_{\alpha}=0 ; \\
g_{\alpha}^{A} \cdot g_{\beta}^{B} \cdot\left(\xi_{A ; B}+\xi_{B ; A}\right)=\varphi \cdot \bar{\partial}_{\Lambda}^{+} g_{\alpha \beta}=0 \Rightarrow \bar{\partial}_{\Lambda}^{+} g_{\alpha \beta}=0 .
\end{gathered}
$$

In the case of the $5 \mathrm{D}$ geodetic equations, the process of the $(4+1)$ splitting one can make the same way. Of course, when one is making this splitting of Equation (4) one 
should take into account the content of Section 2 and the above-mentioned relations. Thus, to obtain Equations (5) and (6) one needs to project firstly Equation (4) with the help of the monad vector $\lambda_{A}$ and then with the help of the metric tensor $g_{A}^{\alpha}$.

The evaluation of the above-mentioned integral of Equation (5) leads one directly to the following exact expression for the effective generalized rest mass:

$$
\hat{m}_{0}=\frac{m_{0}}{\sqrt{1-\hat{u}^{2}}}=\sqrt{m_{0}^{2}+\frac{Q_{0}^{2}}{4 \cdot k_{0} \cdot \varphi^{2}}}=\sqrt{m_{0}^{2}+\frac{n^{2} \cdot \hat{m}_{P l}^{2}}{\varphi^{2}}}
$$

It should be also stressed that Equation (7) has the same form as the corresponding expression in the geodetic equations in a scalar-tensor theory [13], but up to the Lorentz force. Then, in this theory, it was just declared, following Mach's principle that the rest mass must be a certain unknown function of the scalar field $\varphi$ and it should interact with this scalar field through the hypothetical scalar charge $Q_{S c}$. The explicit form of this function remains unknown till now and the scalar charge has not been detected yet and remains a hypothetical quantity. In contrast, Equation (18) provides the definite, single-valued explicit expression for $\hat{m}_{0}$. Note that Equation (18) also agrees with the economy principle of nature: $Q_{0} \equiv Q_{S c}$. Thus, one can believe that rather there is not any scalar charge $Q_{S c}$ in Nature. It means that an electric charge is simultaneously the scalar one. Thus, one can conclude that the neutral particles in the scalar-tensor theory interact neither with a scalar field nor with an electromagnetic one. In an opposite case, one needs to generalize the well-known Equivalence Principle in General Relativity, spreading it also on the scalar field and being under the hypothesis that almost all particles, excepting photon and neutrino, are the composite ones. Here we also should repeat that the relation (18) generalizes the above-mentioned expression for invariant $I$ obtained by L. de Broglie [7].

Expression (18) may be rewritten in a more compact and convenient form by introducing the mass angle:

$$
\chi_{n}=\operatorname{Arsh} \frac{n \cdot \hat{m}_{P l}}{m_{0} \cdot \varphi}: \hat{m}_{0}=m_{0} \cdot \operatorname{ch} \chi_{n} .
$$

Besides, one may represent this expression (18) as a modulus of a complex quantity:

$$
\hat{m}_{0}=\sqrt{\hat{m}_{0 z} \cdot \hat{\bar{m}}_{0 z}}
$$

where $\hat{m}_{0 z}=m_{0}+i \cdot n \cdot \hat{m}_{P l} / \varphi$. It may be written also in the exponential form as $\hat{m}_{0 z}=$ $\hat{m}_{0} \cdot e^{i \cdot \psi_{n}}$, where the phase $\psi_{n}=\arctan \left\{n \cdot \hat{m}_{P l} /\left(m_{0} \cdot \varphi\right)\right\}$. Here $\hat{\bar{m}}_{0 z}=m_{0}-i \cdot n \cdot \hat{m}_{P l} / \varphi$ is a complex conjugate to the $\hat{m}_{0 z}$ quantity. It motivates one to assume in it a possible connection with the quantum properties of the elementary particles. In these terms, one may consider, for example, a photon as a complex null-particle $(0 ; 0)$, a neutrino as a real particle $\left(m_{v} ; 0\right)$, and a hypothetical tachyon as an imaginary particle $\left(0 ; m_{\tau}\right)$ [12]. Thus, one must stress again that it seems to be natural to spread out the equivalence principle of the general relativity for gravitational field additionally also on the scalar gravitational field and therefore, to put forward the hypothesis that almost every verily $5 \mathrm{D}$ particle must contain an electric charge, leastwise in a latent form. Basing on this idea one can also to suppose that the neutral 5D particles must be the composite ones. In this sense, as we believe, there are rather only two verily $4 \mathrm{D}$ particles: the photon and the neutrino. Specifically, the existence of the hypothetical tachyon one can connect just with the fifth coordinate. An additional argument to introduce the effective generalized rest mass is also such a fact that only in this case is it possible to perform the further $(3+1+1)$ splitting of the 5D geodetic Equation (7) with an additional elimination of the time coordinate, using the dyad method $[3,4,11]$. Only in this case, we can obtain the well-known system of the geodetic equations, which is very familiar to the same system that we usually obtain in $4 \mathrm{D}$ theory, making the similar $(3+1)$ splitting of the $4 \mathrm{D}$ geodetic equation with help of the $4 \mathrm{D}$ monad method with the elimination of the $x^{0}$ coordinate but up to the $4 \mathrm{D}$ scalar force. 
Thus, in the 5D case we can obtain the following system from Equation (7), making the further $(3+1+1)$ splitting of it $[2-4,11,12]$ :

$$
\begin{gathered}
m_{0} \cdot \tau_{A} \cdot G^{A}=0 \Rightarrow \frac{D^{+} \hat{m}}{d \tau}=\hat{\bar{p}}^{i} \cdot\left(\bar{F}_{i}-\bar{v}^{k} \cdot \bar{D}_{i k}\right)-\frac{Q_{0}}{c^{2}} \cdot \bar{v}^{i} \cdot \bar{E}_{i}+\frac{Q_{0} \cdot \sqrt{1-\bar{v}^{2}}}{2 \cdot \sqrt{k_{0}} \cdot \varphi} \cdot \bar{\partial}_{\tau}^{+} \hat{m}_{0} \\
-m_{0} \cdot h_{A}^{i} \cdot G^{A}=0 \Rightarrow \\
\Rightarrow \frac{D^{+} \hat{\bar{p}}^{i}}{d \tau}=\frac{Q_{0}}{c^{2}} \cdot\left(\bar{E}^{i}-\bar{v}^{k} \cdot H_{. k}^{i}\right)-\hat{m} \cdot \bar{F}^{i}+2 \cdot \hat{\bar{p}}^{k} \cdot \bar{D}_{k}^{i}+\frac{Q_{0} \cdot \sqrt{1-\bar{v}^{2}}}{2 \cdot \sqrt{k_{0}} \cdot \varphi} \cdot \bar{\partial}^{+i} \hat{m}_{0} .
\end{gathered}
$$

Here $\frac{D^{+}}{d \tau}=\bar{\partial}_{\tau}^{+}+\hat{v} \cdot \bar{\partial}_{\Lambda}^{+}+\bar{v}^{i} \cdot \bar{\nabla}_{i}^{+}$and the indexes $i, j, k, \ldots=1,2,3$.

Specifically, it occurred that it is impossible in this case to perform further $(3+1+1)$ splitting of Equation (7) without introducing an effective generalized rest mass $\hat{m}_{0}$ because in an opposite case Equations (21) and (22) are here coupled so strongly that there is not any possibility to disconnect them. The last terms in Equations (21) and (22) are the components of the scalar $4 \mathrm{D}$ force which are caused by the dependence of the effective generalized rest mass $\hat{m}_{0}$ on the scalar gravitational field. Here we have also put the well-known designations: $\hat{m}=\hat{m}_{0} / \sqrt{1-\bar{v}^{2}}, \bar{v}^{i}=d \bar{x}^{i} / d \tau, \hat{v}=d \lambda / d \tau$. Then, $\hat{p}^{i}=\hat{m} \cdot \bar{v}^{i}, \hat{p}=\hat{m} \cdot \hat{v}=$ $\hat{m}_{0} \cdot \hat{u}$, and $d \tau=c \cdot d t$. It should be also noted that the scalar $4 \mathrm{D}$ force in Equations (21) and (22) vanishes for the zero rest mass particles, as it was predicted in all of the scalar-tensor theories, due to the Lorentz factor $\sqrt{1-\bar{v}^{2}}$ [13].

Note here that the effective generalized rest mass $\hat{m}_{0}$ in the $5 \mathrm{D}$ theory appears just in the same way as the relativistic mass $m=m_{0} / \sqrt{1-\bar{v}^{2}}$ in the $4 \mathrm{D}$ theory:

$$
d l \rightarrow d s \Rightarrow d s^{2}=d \tau^{2}-d l^{2} ; m=m_{0} \cdot \beta ; \bar{v}=d l / d \tau ; d \tau=c \cdot d t,
$$

where the usual $4 \mathrm{D}$ Lorentz factor $\beta=\left(1-\bar{v}^{2}\right)^{-1 / 2}$. Thus, one can believe the $\hat{m}_{0}$ to be, in some sense, the 5D "relativistic" effective generalized rest mass Ref. [14].

It is easy to prove that the same results one can obtain whenever we increase the space dimensions of the space-time: $V_{n} \mapsto V_{n+1}$. As a consequence, one can obtain here the following result: $\hat{m}_{0, n} \mapsto \hat{m}_{0, n+1}$. Thus, following the idea of P. Ehrenfest [15], one can be convinced here that the increase in the dimension adds some new features to the physical nature of the particles and to nature itself.

We can easily bring the geodetic Equation (7) to the following form:

$$
\hat{m}_{0} \cdot \frac{D^{+} \bar{u}^{\alpha}}{d s}=f_{L}^{\alpha}+f_{B D}^{\alpha} .
$$

Here

$$
f_{L}^{\alpha}=\frac{Q_{0}}{c^{2}} \cdot \bar{u}^{\beta} \cdot F_{\beta}^{\alpha}
$$

and

$$
f_{B D}^{\alpha}=-\frac{Q_{0}^{2} \cdot P^{\alpha \beta} \cdot \Phi_{\beta}}{4 \cdot k_{0} \cdot \hat{m}_{0} \cdot \varphi^{2}}
$$

are the $4 \mathrm{D}$ Lorentz force and the $4 \mathrm{D}$ scalar one or, as we have proposed [13], the BranseDicke force. We may leave the decision of this question on the world physical community. Here $P^{\alpha \beta}=g^{\alpha \beta}-\bar{u}^{\alpha} \cdot \bar{u}^{\beta}$ is a tensor of the orthogonal projection upon the direction of the $4 \mathrm{D}$ velocity $\bar{u}^{\alpha}$ and $\Phi_{\beta}=\bar{\nabla}_{\beta}^{+} \ln \varphi$. It should be noted that the $4 \mathrm{D}$ Lorentz force for big masses is equal to zero because it depends on the $Q_{0}$ linearly, but the $4 \mathrm{D}$ Branse-Dicke force depends on the $Q_{0}^{2}$ and, although it is very weak [12], it can be very significant, being accumulated for the big masses. One can call it the big masses effect. Besides, the 4D scalar force is rather also negative and so it may have the repelling property. Thus, it may be one of the reasons for the universe's expansion. I would like to note also again that the expression (18) for the effective generalized rest mass permits us to think that 
an electric charge $e$ superposes simultaneously, as it was mentioned above, the role of the scalar one, so it can explain to us why we could not find this scalar charge so far. Furthermore, one can hope that an interplay between the scalar gravitational field and an electric charge can explain how the dark matter may contribute to the total mass of the $4 \mathrm{D}$ universe, which is immersed, in some sense, into the scalar boson ocean and also, highly likely, to the dark energy of the universe. It should also be added that the scalar gravitational field contributes to the braking (some authors prefer here the German term bremsstrahlung) radiation force [12] and permits one to generalize the concept of the last one: $g^{\alpha}=g_{E}^{\alpha}+g_{S c}^{\alpha}+g_{E S c}^{\alpha}$. Here we should inform that the total braking (bremsstrahlung) radiation force may be represented as a sum of the electromagnetic, scalar, and mixed parts, where the first one has the following well-known form:

$$
g_{E}^{\alpha}=\frac{2 \cdot e^{3} \bar{u}^{\gamma}}{3 \cdot \hat{m}_{0} \cdot c^{3}} \cdot\left(\bar{u}^{\beta} \cdot \bar{\nabla}_{\gamma}^{+} F_{\beta}^{\alpha}+\frac{e \cdot P^{\alpha \delta}}{\hat{m}_{0} \cdot c^{2}} \cdot F_{\beta \delta} \cdot F_{\gamma}^{\beta}\right)
$$

and the second one has the form as below:

$$
g_{S c}^{\alpha}=-\frac{e^{4} \cdot P^{\alpha \beta} \cdot \bar{u}^{\gamma}}{6 \cdot c \cdot k_{0} \cdot \varphi^{2} \cdot \hat{m}_{0}^{2}} \cdot\left(\bar{\nabla}_{\gamma}^{+} \Phi_{\beta}-2 \Phi_{\beta} \cdot \Phi_{\gamma}+\frac{3 \cdot e^{2} \cdot \Phi_{\beta} \cdot \Phi_{\gamma}}{4 \cdot k_{0} \cdot \varphi^{2} \cdot \hat{m}_{0}^{2}}\right) .
$$

Lastly, the third one has the next form:

$$
g_{E S c}^{\alpha}=-\frac{e^{5}}{6 \cdot c^{3} \cdot k_{0} \cdot \varphi^{2} \cdot \hat{m}_{0}^{3}} \cdot\left(g^{\beta \delta} \cdot P^{\alpha \gamma}-3 \cdot g^{\alpha \gamma} \cdot \bar{u}^{\beta} \cdot \bar{u}^{\delta}\right) \cdot F_{\beta \gamma} \cdot \Phi_{\delta} .
$$

(See [13] for more details). Of course, the electromagnetic part is here the most significant.

\section{The 5D Ricci Identities and Some Problems of the Physics, Astrophysics, and Cosmology}

We should remember some interesting and perspicacious ideas of the scientific classics about the connections between world geometry and physical interactions. Following one of the greatest mathematicians of the 19th century, W.K. Clifford [16], let us consider the Ricci identities well-known in Riemannian geometry in the case of the 5D theory:

$$
R_{(B C D)}^{A} \equiv R_{\cdot B C D}^{A}+R_{\cdot D B C}^{A}+R_{\cdot C D B}^{A}=0
$$

The (4+1) splitting of the $V_{5}$ (see above the analogous procedures in relations (5)(7)) gives us the following relations between the $4 \mathrm{D}$ physical-geometrical quantities [12], starting with the $4 \mathrm{D}$ Ricci identities:

$$
g_{A}^{\alpha} \cdot g_{\beta}^{B} \cdot g_{\gamma}^{C} \cdot g_{\delta}^{D} \cdot R_{(B C D)}^{A} \equiv R_{(\beta \gamma \delta)}^{\alpha} \equiv R_{\cdot \beta \gamma \delta}^{\alpha}+R_{\cdot \delta \beta \gamma}^{\alpha}+R_{\cdot \gamma \delta \beta}^{\alpha}=0
$$

It means that the $4 \mathrm{D}$ world also has Riemannian geometry. Then, we can obtain the following very important connections between the electromagnetic tensor and the curls of the scalar gravitational field's gradients. It gives us a very specific "trigger" of the changing electromagnetic process:

$$
\begin{gathered}
\lambda_{A} \cdot g_{\alpha}^{B} \cdot g_{\beta}^{C} \cdot g_{\gamma}^{D} \cdot R_{(B C D)}^{A}=0 \Rightarrow R_{(\beta \gamma \delta)}^{\alpha} \equiv F_{(\alpha \beta ; \gamma)}+2 \cdot F_{(\alpha \beta} \cdot \Phi_{\gamma)}=0 ; \\
\lambda_{A} \cdot \lambda^{D} \cdot g_{\alpha}^{B} \cdot g_{\beta}^{C} \cdot R_{(B C D)}^{A}=0 \Rightarrow \frac{2 \cdot \sqrt{k_{0}}}{c^{2}} \cdot F_{\alpha \beta} \cdot \bar{\partial}_{\Lambda}^{+} \varphi=\Phi_{\alpha ; \beta}-\Phi_{\beta ; \alpha} \equiv M_{\alpha \beta} .
\end{gathered}
$$

Here we have introduced the curls of the scalar gravitational field's gradients $M_{\alpha \beta}$, which one may consider as the quasiparticles of the soliton-type or magnetic monopoletype. These quasiparticles, in some sense, are similar to solitons $[17,18]$. Importantly that the imposition of the cylindricity condition gives rise to the vanishing of these scalar curls, since only in this case the necessary and sufficient condition for the scalar gravita- 
tional field to be laminar may be satisfied (it is, as well-known, the following condition: $\left.\Phi_{\alpha ; \beta}=\Phi_{\beta ; \alpha} \Rightarrow M_{\alpha \beta} \equiv 0\right)$.

At last, making use of Equations (31) and (32), one can obtain the first pair of the Maxwell equations with a nonzero r.h.s. of the magnetic monopole-type, namely:

$$
F_{(\alpha \beta ; \gamma)} \equiv F_{\alpha \beta ; \gamma}+F_{\gamma \alpha ; \beta}+F_{\beta \gamma ; \alpha}=\frac{2}{\sqrt{\bar{\kappa}_{0}} \cdot \bar{\partial}_{\Lambda}^{+} \varphi} \cdot\left|\begin{array}{ccc}
\Phi_{\alpha} & \bar{\nabla}_{\alpha}^{+} & \Phi_{\alpha} \\
\Phi_{\beta} & \bar{\nabla}_{\beta}^{+} & \Phi_{\beta} \\
\Phi_{\gamma} & \bar{\nabla}_{\gamma}^{+} & \Phi_{\gamma}
\end{array}\right| .
$$

The imposition of the cylindricity condition here also gives rise to the vanishing of the r.h.s of Equation (31), which brings this equation to the conventional form of the first pair of the Maxwell equations:

$$
F_{(\alpha \beta ; \gamma)} \equiv F_{\alpha \beta ; \gamma}+F_{\gamma \alpha ; \beta}+F_{\beta \gamma ; \alpha}=0 .
$$

Thus, one can conclude that the first pair of the Maxwell equations is obliged to the Riemannian structure of the $V_{5}$ and has another origin than the second one, which we have obtained from the $5 \mathrm{D}$ variation principle [4,19]. The r.h.s. in Equation (33) one can rewrite employing the above-mentioned curls $M_{\alpha \beta}$ in the following form:

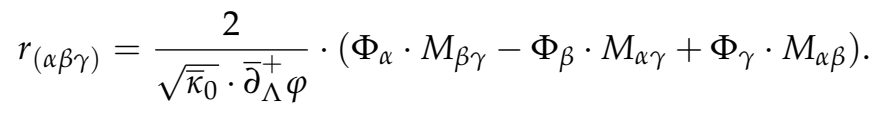

This gives us the possibility to represent the second pair of the Maxwell equations, which we have obtained, as we have mentioned above, from the 5D variation principle [4,19], with the same monopole-type r.h.s., if we believe that this r.h.s. is the linear combination of the magnetic monopole-type currents. With this purpose, we will write down the mixed, $x^{5}$ and $V_{4}$ projected, 5D field equations with the energy-momentum tensor of the $5 \mathrm{D}$ dust as a r.h.s. $[4,19]$ :

$$
\bar{\nabla}_{v}^{+}\left(\varphi^{3} \cdot F^{\mu v}\right)=-\frac{8 \cdot \pi}{c^{2}} \cdot \sqrt{k_{0}} \cdot \varphi \cdot Q_{5}^{\mu}
$$

Here the energy-momentum tensor of the 5D dust has the following form $[4,19]$ :

$$
Q^{A B}=\mu_{0} \cdot c \cdot \frac{d x^{A}}{d I} \cdot \frac{d x^{B}}{d \tau}
$$

where $\mu_{0}$ is a matter density. However, before the cylindricity condition is imposed, one may easily bring Equation (36) to the following form, casting temporarily aside the r.h.s. in Equation (36) and using Equation (32):

$$
\bar{\nabla}_{v}^{+} F^{\mu v}=-\frac{3 \cdot c^{2} \cdot \Phi_{v} \cdot M^{\mu v}}{2 \cdot \sqrt{k_{0}} \cdot \bar{\partial}_{\Lambda}^{+} \varphi}=-\frac{4 \cdot \pi}{c} \cdot \bar{j}_{m}^{\mu} .
$$

We can interpret here that the r.h.s. in (38) as a magnetic monopole-type current density, where we can believe that curl $M_{\mu v}$ corresponds, for example, to the "north" particle of the magnetic monopole-type and $M_{v \mu}$ corresponds to the "south" one, or vice versa:

$$
j_{m}^{\mu}=\frac{3 \cdot c^{3} \cdot \Phi_{v} \cdot M^{\mu v}}{8 \cdot \pi \cdot \sqrt{k_{0}} \cdot \bar{\partial}_{\Lambda}^{+} \varphi}
$$

Let us call them $n$-monopole and s-monopole. Thus, we believe that there exist two types of the magnetic monopole "charges": $n$ and $s$ ones.

Finally, we can establish that just after we have imposed the cylindricity condition. As a result, the curls of the scalar gravitational field disappear and then Equation (5) gives us an integral of the movement along the fifth coordinate connected with an electric charge 
of the 5D test particle, so thus the energy-momentum tensor of the 5D dust (the r.h.s. in Equation (36)) easily transforms in the second pair of the Maxwell equations and gives us the new r.h.s. of the conventional type as follows below [4,19]:

$$
\nabla_{v}^{+} F^{\mu v}=-\frac{4 \cdot \pi}{c \cdot \varphi^{2}} \cdot j_{e}^{\mu}
$$

Here $j_{e}^{\mu}=\rho_{0} \cdot \bar{v}^{\mu}$ is the $4 \mathrm{D}$ electric current ( $\rho_{0}$ is an electric charge density). We have used here the relation (36) and then we have also used the evident relation: $\rho_{0}=\mu_{0} \cdot Q_{0} / m_{0}$.

One can join Equations (38) and (40) by using the Heaviside's unit-jump-function $H(t)$ as it is defined below:

$$
H(t)=0 \text { at } t<0 \text { and } H(t)=1 \text { at } t \geq 0 .
$$

Thus, one can write down now the second pair of the Maxwell equations (I have also proposed to call them the Maxwell-Dirac equations [12]) as follows below:

$$
\bar{\nabla}_{v}^{+} F^{\mu v}=-\frac{4 \cdot \pi}{c} \cdot\left[H\left(t_{0}-t\right) \cdot \bar{j}_{m}^{\mu}+H\left(t-t_{0}\right) \cdot \varphi^{-2} \cdot \bar{j}_{e}^{\mu}\right] .
$$

Here $t_{0}$ is the moment of the time, when the $x^{5}$ cylindricity condition was established. The first pair of the Maxwell-Dirac equations one can also write down employing the Heaviside function:

$$
F_{(\alpha \beta ; \gamma)} \equiv F_{\alpha \beta ; \gamma}+F_{\gamma \alpha ; \beta}+F_{\beta \gamma ; \alpha}=H\left(t_{0}-t\right) \cdot r_{(\alpha \beta \gamma)}
$$

The analysis of these results leads us to the fundamental idea about the evolution of the $4 \mathrm{D}$ Universe. The process of the transition to the cylindrical symmetry condition in the 5D Universe $\left(V_{5} \rightarrow V_{4}\right)$ seems to be, in some sense, very familiar with the second-order phase transition in helium. As is well-known, the cooling of the helium leads to the secondorder phase transition [20] of the last and this process attends with the disappearance of the collective curl excitations (rotons) and, as a result of the transition to the laminar state, with the appearance of the phonons. Then, we may hypothesize that here we have some kind of a second-order phase transition in cosmology. Let us call it the topological one [18]. It is quite possible that this phase transition is connected with the cooling of the expanding universe and leads to the more stable state of the universe's aggregation. Note also that work [21] obtained the energetical spectrum of some kind of scalar gravitational field that looks very similar to the energetical spectrum of helium. Thus, one can suppose that, as a result of this phase transition, we have obtained some kind of a superfluid state of the scalar matter and it may accelerate the expansion of the 4D universe [18]. This acceleration, as well-known, was discovered more than twenty years ago with help of the observations after the type Ia supernovas' explosions. Moreover, we may add that this transition and further compaction of the fifth coordinate is possibly caused by the Casimir effect [22]. Besides, one can assume that in modern time we are not able to find these magnetic monopoles in the $4 \mathrm{D}$ universe, maybe only a few of the old ones [12,18]. In addition, one can hypothesize, based on the expression (18), that the effective rest mass of the particles, being dependent on the scalar $\varphi=\eta(t)$, which in turn is connected with the 5D metric $G_{55}$ (See above Section 2, formula (1)), possibly slightly (as an adiabatic invariant), varies with the cosmological time $t$. In work [23] the authors have considered a 5D metric of the Kasner type, which depends on the cosmological time. Then, maybe one can estimate the age of the 4D universe, comparing the values of the elementary particles' masses at different moments of the cosmological time $t$.

Thus, hopefully, one day we will be able, among many other questions, to answer the age-old question: "Where has the magnetic monopole gone?" [24]. 


\section{Discussion}

This article was written because over a long time the author has accumulated many results in the 5D theory of the Kaluza type, which has been almost forgotten during the last fifty years. If one goes beyond the 5D optics, certain new and very significant properties of the matter and $4 \mathrm{D}$ Universe may come into being. Following this approach, the author succeeded to generalize the rest mass concept following the ideas of L. de Broglie and has understood more deeply the quantum nature of the matter. Furthermore, the structure of the generalized rest mass gave the possibility of believing that this mass may have a complex structure. It has led to the idea about connection between the 5D theory and particles' structure, including the tachyons problem. The implementation of the monad method to the $(4+1)$ splitting of the 5D Ricci identities makes it possible to understand how the Riemannian structure of the world affects its physical properties. It permits one to approach closer to the understanding of the magnetic monopole's problem and the difference in the origins of the first and the second pairs of the Maxwell equations, and at the same time the surprising connections between them. The obtained results also provide new insight into the mechanism of the expansion of the $4 \mathrm{D}$ universe and its acceleration. The author believes that the application of the developed approach extends far beyond the specific problems discussed in the present paper. Its generalization beyond the $5 \mathrm{D}$ case may be also worthwhile and fruitful.

\section{Summary of the Main Results}

I have reviewed the old and modern publications on 5D theory, and have not found any papers with the same results. The most relevant found publications are the articles by $\mathrm{L}$. de Broglie (See Ref. [7] in my references) and by T.X. Zhang (See Ref. [9] in my references), whose results are similar to a certain part of my results. However, the results presented in my MS are more general and broader than those obtained by L. de Broglie and T.X. Zhang. The corresponding discussion is possible to find in the last version of the MS, see p. 6 .

To emphasize the novel points of my accomplishments, I have added a summary of the main old and new results in my MS just after point 5 (Discussion). Specifically, the new rest mass concept was introduced in a natural way in the process of the (4+1) splitting of the $\mathrm{V}_{5}$ by using the monad method (See the Equations (5)-(7) and Appendix A in my MS). It should be noted that this problem was first put by L. de Broglie (See his private result in (Ref. [7], Equation (41)) and the following discussion on pages 5 and 6 of my MS). Then, this problem was touched on in scalar-tensor theories based on Mach's Principle (See Ref. [13] and discussion on p. 7 of my MS). All of that has led to the possible understanding of the problem with the mysterious scalar charge's absence. In addition, it gave rise to a compact and physically clear formula (See formula (18) in my MS) for this before-unknown generalized mass as a function of the scalar field in the case of the scalar-tensor theories.

Besides, it permits one to introduce the compact formula with mass angle. It should be added that this concept leads one to the possible conclusion about the complex structure of the particle's rest mass. It gives an additional idea about the possible connection between the fifth coordinate $x^{5}$ and the hypothetical tachyon's existence. This hypothesis may help to obtain a better understanding how the $5 \mathrm{D}$ theory is connected with the quantum theory. The structure of the generalized rest mass gives rise to the hypothesis that it may contribute to the dark matter and dark energy (See p. 10). Here was introduced the 4D scalar gravitational force, which may be one of the reasons for the universe's expansion (See p. 9).

The next group of results I obtained after $(4+1)$ splitting of the well known 5D Ricci identities. It gave certain relations between the different $4 \mathrm{D}$ physicogeometrical quantities of the electromagnetic and also scalar gravitational fields (See Equations (30)-(32) in my MS).

Finally, one can obtain the first pair of the Maxwell equations with the nonzero r.h.s. that vanishes after imposition of the $x^{5}$ cylindricity conditions (See Equation (33) in my MS). This leads to the idea about the Dirac's monopole. It has been noted that this transition 
seems to be very familiar to the second-order transition in helium (See the discussion on pp. 11 and 12 of my MS). The latter permits the idea that this transition leads to the superfluidity of scalar matter, which may accelerate the universe's expansion (See the discussion on pp. 11 and 13). All of that have led to the hypothesis about the two ages in the universe's evolution: the age of the magnetic monopole and age of the electron. Thus, one can able to generalize two pairs of the Maxwell equations and establish the difference in their origin together with the connection between them.

\section{Conclusions}

The author would like to express the hope that these results may open a new age in the investigations of the 5D theory, which was appreciated by one of the greatest physicists, L. de Broglie, as a preferable one, and will attract more attention of the world's physics community. It should be also noted that the possible discovering of the fifth force with help of the circular accelerator in Batavia may inspire the new generations of researchers and stimulate their interest to investigate deeper the 5D theory.

Funding: This research received no external funding.

Acknowledgments: The author would like to thank with great pleasure Yu.S. Vladimirov, A.V. Solovyov, M.I. Tribelsky, K.G. Chetyrkin, K.A. Bronnikov, V.A. Rubakov, and V.A. Berezin for useful discussions. The author is also grateful to A.V. Solovyov, O.P. Goldshteyn, and I.B. Aliyev for the valuable help in the manuscript preparation.

Conflicts of Interest: The author declares no conflict of interest.

\section{Appendix A}

Here we consider in detail several examples of the obtaining of the above-mentioned relations. Let us start with the Killing Equations (A1)-(A4):

$$
\lambda^{A} \cdot \lambda^{B} \cdot L_{\xi} G_{A B}=\lambda^{A} \cdot \lambda^{B} \cdot\left(\xi_{A ; B}+\xi_{B ; A}\right)=-2 \cdot \bar{\partial}_{\Lambda}^{+} \varphi \Rightarrow \bar{\partial}_{\Lambda}^{+} \varphi=0 ;
$$

The detailed calculations give us the same result as in relation (15):

$$
\begin{gathered}
2 \cdot \lambda^{A} \cdot \lambda^{B} \cdot\left(\varphi \cdot \lambda_{A}\right)_{; B}=2 \cdot \varphi^{-2} \cdot\left(-\partial_{5} \varphi^{2}-P_{55,5} \cdot \varphi^{-1} \cdot \varphi\right)= \\
-4 \cdot \varphi^{-1} \cdot \varphi_{, 5}-\varphi^{-2} \cdot\left(2 \cdot G_{55,5}-G_{55,5}\right)=-4 \cdot \bar{\partial}_{\Lambda}^{+} \varphi+2 \cdot \bar{\partial}_{\Lambda}^{+} \varphi=-2 \cdot \bar{\partial}_{\Lambda}^{+} \varphi=0 ; \\
\lambda^{A} \cdot g_{\alpha}^{B} \cdot\left(\xi_{A ; B}+\xi_{B ; A}\right)=\varphi^{2} \cdot \bar{\partial}_{\Lambda}^{+}\left(\lambda_{\alpha} / \varphi\right)=\frac{2}{c^{2}} \sqrt{k_{0}} \cdot \varphi^{2} \cdot \bar{\partial}_{\Lambda}^{+} A_{\alpha}=0 \Rightarrow \bar{\partial}_{\Lambda}^{+} A_{\alpha}=0 ;
\end{gathered}
$$

and one can obtain an analogical result for relation (16) as follows below:

$$
\begin{gathered}
\lambda^{A} \cdot g_{\alpha}^{B} \cdot\left[\partial_{A}\left(\varphi \cdot \lambda_{B}\right)+\partial_{B}\left(\varphi \cdot \lambda_{A}\right)-2 \cdot P_{A B, C} \cdot \varphi \cdot \lambda^{C}\right]=\varphi \cdot\left(g_{\alpha}^{A} \cdot \bar{\partial}_{\Lambda}^{+} \lambda_{A}-2 \cdot \Phi_{\alpha}\right)- \\
-2 \cdot \varphi^{-1} \cdot g_{\alpha}^{A} \cdot P_{5 A, 5}=\varphi^{2} \cdot \bar{\partial}_{\Lambda}^{+}\left(\lambda_{\alpha} / \varphi\right)-2 \cdot \varphi \cdot \Phi_{\alpha}-\varphi^{-1} \cdot g_{\alpha}^{A} \cdot \partial_{A} G_{55}= \\
\varphi^{2} \cdot \bar{\partial}_{\Lambda}^{+}\left(\lambda_{\alpha} / \varphi\right)-2 \cdot \varphi \cdot \Phi_{\alpha}+2 \cdot \varphi \cdot \Phi_{\alpha}=\frac{2 \cdot \sqrt{k_{0}}}{c^{2}} \cdot \bar{\partial}_{\Lambda}^{+} A_{\alpha}=0 \Rightarrow \bar{\partial}_{\Lambda}^{+} A_{\alpha}=0 .
\end{gathered}
$$

It should be reminded here that $G_{55}=-\varphi^{2}$ and $A_{\alpha}$ is a $4 \mathrm{D}$ vector-potential of the electromagnetic field.

$$
g_{\alpha}^{A} \cdot g_{\beta}^{B} \cdot\left(\xi_{A ; B}+\xi_{B ; A}\right)=\varphi \cdot \bar{\partial}_{\Lambda}^{+} g_{\alpha \beta}=0 \Rightarrow \bar{\partial}_{\Lambda}^{+} g_{\alpha \beta}=0 .
$$

Finally, one can also obtain the above-mentioned relation (17) as follows below:

$$
g_{\alpha}^{A} \cdot g_{\beta}^{B} \cdot\left[\partial_{A}\left(\varphi \cdot \lambda_{B}\right)+\partial_{B}\left(\varphi \cdot \lambda_{A}\right)-2 \cdot P_{A B, C} \cdot \varphi \cdot \lambda^{C}\right]=g_{\alpha}^{A} \cdot \bar{\partial}_{\beta}^{+}\left(\varphi \cdot \lambda_{A}\right)+g_{\beta}^{B} \cdot \bar{\partial}_{\alpha}^{+}\left(\varphi \cdot \lambda_{B}\right)-
$$




$$
\begin{gathered}
-g_{\beta}^{B} \bar{\partial}_{\alpha}^{+}\left(\varphi \cdot \lambda_{B}\right)-g_{\alpha}^{A} \cdot \bar{\partial}_{\beta}^{+}\left(\varphi \cdot \lambda_{A}\right)+\varphi \cdot g_{\alpha}^{A} \cdot g_{\beta}^{B} \cdot \bar{\partial}_{\Lambda}^{+}\left(g_{A B}-\lambda_{A} \cdot \lambda_{B}\right)=\varphi \cdot \bar{\partial}_{\Lambda}^{+} g_{\alpha \beta}=0 \\
\Rightarrow \bar{\partial}_{\Lambda}^{+} g_{\alpha \beta}=0 .
\end{gathered}
$$

Now we will pay attention to the geodetic Equations (5) and (6). It's suitable to represent these calculations divided into two parts. For the first part of relation (5), one can obtain:

$$
\begin{gathered}
m_{0} \cdot \lambda_{A} \cdot \frac{d^{2} x^{A}}{d I^{2}}=\hat{\beta} \cdot \lambda_{A} \cdot \frac{d}{d s}\left(m_{0} \cdot \hat{\beta} \cdot \frac{d x^{A}}{d s}\right)=\hat{\beta} \cdot \lambda_{A} \cdot \frac{d}{d s}\left(\hat{m}_{0} \cdot u^{A}\right)=\hat{\beta} \cdot \lambda_{A} \cdot\left(\hat{m}_{0} \cdot \frac{d u^{A}}{d s}+u^{A} \cdot \frac{d \hat{m}_{0}}{d s}\right)= \\
=\hat{\beta} \cdot\left(\hat{m}_{0} \cdot \lambda_{A} \cdot \frac{d u^{A}}{d s}+\lambda_{A} \cdot u^{A} \cdot \frac{d \hat{m}_{0}}{d s}\right)=\hat{\beta} \cdot\left[\hat{m}_{0} \cdot\left(\frac{d \hat{u}}{d s}-u^{A} \cdot \frac{d \lambda_{A}}{d s}\right)+\hat{u} \cdot \frac{d \hat{m}_{0}}{d s}\right]= \\
=\hat{\beta} \cdot\left(\frac{d \hat{p}}{d s}-\hat{m}_{0} \cdot u^{A} \cdot \frac{d \lambda_{A}}{d s}\right) ;
\end{gathered}
$$

here $\lambda_{A} \cdot u^{A}=\hat{u}$; and $\hat{m}_{0} \cdot \hat{u}=\hat{p}$; then, for the second part of the relation (5), one can also obtain:

$$
\begin{aligned}
& m_{0} \cdot \lambda_{A} \cdot P_{B C}^{A} \cdot \frac{d x^{B}}{d I} \cdot \frac{d x^{C}}{d I}=\hat{m}_{0} \cdot \hat{\beta} \cdot \lambda^{A} \cdot P_{B C, A} \cdot u^{B} \cdot u^{C}=\hat{\beta} \cdot \hat{m}_{0} \cdot \frac{P_{B C, 5}}{\varphi} \cdot u^{B} \cdot u^{C}= \\
& =\frac{\hat{\beta} \cdot \hat{m}_{0}}{2 \cdot \varphi} \cdot\left(2 \cdot \partial_{B} G_{C 5}-\partial_{5} G_{B C}\right) \cdot u^{B} \cdot u^{C}= \\
& \hat{\beta} \cdot \hat{m}_{0} \cdot\left[\frac{\partial_{B}\left(\varphi \cdot \lambda_{C}\right)}{\varphi}-\frac{\partial_{\Lambda}^{+}\left(g_{B C}-\lambda_{B} \cdot \lambda_{C}\right)}{2}\right] \cdot u^{B} \cdot u^{C}= \\
& =\hat{\beta} \cdot \hat{m}_{0} \cdot\left(u^{A} \cdot \frac{d \lambda_{A}}{d s}+\hat{u} \cdot \frac{d \ln \varphi}{d s}+\hat{u} \cdot u^{A} \cdot \bar{\partial}_{\Lambda}^{+} \lambda_{A}-\frac{1}{2} \cdot \bar{u}^{\alpha} \cdot \bar{u}^{\beta} \cdot \bar{\partial}_{\Lambda}^{+} g_{\alpha \beta}\right) .
\end{aligned}
$$

Adding two parts of the relation (5), one can easily obtain the following result:

$$
\begin{aligned}
& m_{0} \cdot \lambda_{A} \cdot G^{A}=\hat{\beta} \cdot\left(\frac{d \hat{p}}{d s}-\hat{m}_{0} \cdot u^{A} \cdot \frac{d \lambda_{A}}{d s}+\hat{m}_{0} \cdot u^{A} \cdot \frac{d \lambda_{A}}{d s}+\hat{p} \cdot \frac{d \ln \varphi}{d s}+\frac{0}{1}\right)= \\
& \frac{\hat{\beta}}{\varphi} \cdot \frac{d}{d s}(\varphi \cdot \hat{p})=0 .
\end{aligned}
$$

Here the last term within the brackets means, as a certain symbol, the sum of the next two quantities below, which are both equal to zero because of the cylindricity condition relative to the fifth coordinate:

$$
\hat{p} \cdot u^{A} \cdot \bar{\partial}_{\Lambda}^{+} \lambda_{A}=0 ; \frac{1}{2} \cdot \bar{p}^{\alpha} \cdot \bar{u}^{\beta} \cdot \bar{\partial}_{\Lambda}^{+} g_{\alpha \beta}=0 \Rightarrow \frac{0}{1}
$$

Finally, one can consider and obtain by the same way the relation (6) as follows:

$$
\begin{gathered}
m_{0} \cdot g_{A}^{\alpha} \cdot \frac{d^{2} x^{A}}{d I^{2}}=\hat{\beta} \cdot g_{A}^{\alpha} \cdot \frac{d}{d s}\left(m_{0} \cdot \hat{\beta} \cdot u^{A}\right)=\hat{\beta} \cdot g_{A}^{\alpha} \cdot \frac{d}{d s}\left(\hat{m}_{0} \cdot u^{A}\right)=\hat{\beta} \cdot \frac{d \bar{p}^{\alpha}}{d s} ; \\
m_{0} \cdot g_{A}^{\alpha} \cdot P_{B C}^{A} \cdot \frac{d x^{B}}{d I} \cdot \frac{d x^{C}}{d I}=m_{0} \cdot \hat{\beta}^{2} \cdot g^{\alpha A} \cdot P_{B C, A} \cdot u^{B} \cdot u^{C}=\hat{m}_{0} \cdot \hat{\beta} \cdot g^{\alpha A} \cdot\left(\partial_{B} G_{C A}-\partial_{A} G_{B C} / 2\right) \cdot u^{B} \cdot u^{C}= \\
=\hat{m}_{0} \cdot \hat{\beta} \cdot g^{\alpha A} \cdot\left[\partial_{B}\left(g_{C A}-\lambda_{C} \cdot \lambda_{A}\right)-\partial_{A}\left(g_{B C}-\lambda_{B} \cdot \lambda_{C}\right) / 2\right] \cdot u^{B} \cdot u^{C}= \\
=\hat{m}_{0} \cdot \hat{\beta} \cdot\left(g^{\alpha \beta} \cdot u^{B} \cdot \bar{u}^{\gamma} \cdot \partial_{B} g_{\beta \gamma}-\hat{u} \cdot u^{B} \cdot g^{\alpha A} \cdot \partial_{B} \lambda_{A}-\bar{u}^{\beta} \cdot \bar{u}^{\gamma} \cdot \bar{\partial}^{+\alpha} g_{\beta \gamma} / 2+\hat{u} \cdot u^{A} \cdot \bar{\partial}^{+\alpha} \lambda_{A}\right)= \\
=\hat{m}_{0} \cdot \hat{\beta} \cdot\left[g^{\alpha \beta} \cdot \bar{u}^{\gamma} \cdot \bar{u}^{\delta} \cdot\left(\bar{\partial}_{\gamma}^{+} g_{\beta \delta}+\bar{\partial}_{\delta}^{+} g_{\beta \gamma}-\bar{\partial}_{\beta}^{+} g_{\gamma \delta}\right) / 2-\hat{u} \cdot \bar{u}^{\beta} \cdot g^{\alpha A} \cdot \bar{\partial}_{\beta}^{+} \lambda_{A}+\hat{u} \cdot g^{\alpha \beta} \cdot u^{A} \cdot \bar{\partial}_{\beta}^{+} \lambda_{A}\right]= \\
=\hat{m}_{0} \cdot \hat{\beta} \cdot\left(\hat{\Gamma}_{\beta \gamma}^{\alpha} \cdot \bar{u}^{\beta} \cdot \bar{u}^{\gamma}-\frac{2 \cdot \sqrt{k_{0}}}{c^{2}} \cdot \varphi \cdot \hat{u} \cdot \bar{u}^{\beta} \cdot F_{\beta}^{\alpha}+\hat{u}^{2} \cdot \Phi^{\alpha}\right) ;
\end{gathered}
$$

Here, we have used the following evident relations:

$$
g^{\alpha A} \cdot \bar{\partial}_{\beta}^{+} \lambda_{A}=\varphi \cdot \bar{\partial}_{\beta}^{+}\left(\frac{\lambda_{\alpha}}{\varphi}\right)=\varphi \cdot \frac{2 \cdot \sqrt{k_{0}}}{c^{2}} \cdot \bar{\partial}_{\beta}^{+} A_{\alpha} ;
$$




$$
u^{A} \cdot \bar{\partial}^{+\alpha} \lambda_{A}=\varphi \cdot \bar{u}^{\beta} \cdot \bar{\partial}^{+\alpha}\left(\frac{\lambda_{\beta}}{\varphi}\right)+\hat{u} \cdot \Phi^{\alpha}=\varphi \cdot \frac{2 \cdot \sqrt{k_{0}}}{c^{2}} \cdot \bar{\partial}^{+\alpha} A_{\beta}+\hat{u} \cdot \Phi^{\alpha} .
$$

Then one can add these two parts and obtain relation (6) as follows below:

$$
m_{0} \cdot g_{A}^{\alpha} \cdot G^{A}=\hat{\beta} \cdot\left(\frac{D^{+} \bar{p}^{\alpha}}{d s}-\frac{2 \cdot \sqrt{k_{0}}}{c^{2}} \cdot \varphi \cdot \hat{p} \cdot \bar{u}^{\beta} \cdot F_{\beta}^{\alpha}-\bar{\nabla}^{+\alpha} \hat{m}_{0}\right)=0 .
$$

It should be noted that here we have used relations (5) and (18). Then, it helped us to obtain one more evident and very important relations as follows below:

$$
\hat{m}_{0} \cdot \hat{u}^{2} \cdot \Phi^{\alpha}=\left(\frac{n \cdot \hat{m}_{P l}}{\varphi}\right)^{2} \cdot \frac{\Phi^{\alpha}}{\hat{m}_{0}}=-\bar{\nabla}^{+\alpha} \hat{m}_{0} .
$$

For more information and more samples of the calculations in 5D theory, and more references, see also in [1,4]. The author would like to hope that these samples of the calculations with help of the monad method in 5D theory will bring the readers better understanding of the obtained results.

\section{Note}

1 "If one can understand how the constants $e, m_{0}, c, \hbar$ and $k_{0}$ may be interpreted conspicuously in the 5D wave equation, then one will be able to discover the most mysterious secrets of nature."

\section{References}

1. Vladimirov, Y.S. Classical Gravity Theory; Krasand: Moscow, Russia, 2018; pp. 184-207.

2. Aliyev, B.G. Motion equations in the 5D unified field theory. In Abstracts of the 6th International Conference on Gravitation and Relativity Gravitation; GDR: Jena, Germany, 1980; Volume 3, pp. 679-680.

3. Aliyev, B.G. 5D theory of the scalar-tensor gravitation and electromagnetism in dyad form. In Problems of the Theory of Gravitation and Elementary Particles; Stanyuckovich, K.P., Ed.; Atomizdat: Moscow, Russia, 1979; pp. 141-149.

4. Aliyev, B.G. Monad and Dyad Methods in Some Problems of the General Relativity and its Modifications. Ph.D. Thesis, MSU, Moscow, Russia, 1982.

5. Fock, V.A. Über die invariante Form der Wellen-und der Bewegungsgleichungen für einen geladenen Massenpunkt. Z. Phys. 1926, 39, 226-232. [CrossRef]

6. Klein, O. Quantentheorie und fünfdimensionale Relativitätstheorie. Z. Phys. 1926, 37, 895. [CrossRef]

7. De Broglie, L. L’Univers à cinq dimensions et la mécanique ondulatoire. J. Phys. Radium 1927, 8, 65-73. [CrossRef]

8. Rumer, Y.B. Investigations on 5D Optics; State Publishing House of the Technical and Theoretical Literature: Moscow, Russia, 1956.

9. Zhang, T.X. The 5D Fully-Covariant Theory of Gravitation and Its Astrophysical Applications. Galaxies 2015, 3, 18-51. [CrossRef]

10. Bergmann, P.G. Introduction to the Theory of Relativity. In With a Foreword by Albert Einstein; Prentice Hall: New York, NY, USA, 1942.

11. Aliyev, B.G. The behavior of the charged particles in 5D gravity theory. In The Modern Problems of the General Relativity Theory; Physical Institute of the Byelorussian Academy of Sciences: Minsk, Byelorussia, 1979; pp. 154-160.

12. Aliyev, B.G. The effective rest mass concept and magnetic monopole problem in 5D Theory. In Proceedings of the ICGAC-12, Gravity, Astrophysical and Cosmology, Moscow, Russia, 28 June-4 July 2015; Melnikov, V., Hsu, J.P., Eds.; World Scientific: Singapore, 2016; pp. 321-326.

13. Dicke, R.H. Many-sided Mach. In Gravitation and Relativity; Chiu, H.Y., Hoffmann, W.F., Eds.; W.A. Benjamin, Inc.: New York, NY, USA; Amsterdam, The Netherlands, 1964.

14. Aliyev, B.G. The rest mass concept and some problems of Cosmology in 5D Theory. In Abstracts of the RUSGRAV-16; BFU named I. Kant: Kaliningrad, Russia, 2017; p. 91.

15. Ehrenfest, P. In what way does it become manifest in the fundamental laws of Physics that space has three dimensions. Proc. Amst. Acad. 1917, 20, 200.

16. Clifford, W.K. Mathematical Papers; MacMillan: New York, NY, USA; London, UK, 1968.

17. Gross, D.; Perry, M. Magnetic monopoles in Kaluza-Klein theories. Nucl. Phys. 1983, 226, 29. [CrossRef]

18. Aliyev, B.G. The solitons and the topological second-order transition in 5D Theory. In Abstracts of the RUSGRAV-16; BFU named I. Kant: Kaliningrad, Russia, 2017; p. 91.

19. Aliyev, B.G. On the energy-momentum tensor of the 5D dust. In Abstracts of the Reports of the Int. School-Seminar Multidimensional Gravity and Cosmology; RGA: Moscow, Russia, 1994; p. 1.

20. Landau, L.D.; Lifshitz, E.M. The Classical Theory of Fields, 3rd ed.; Pergamon Press: Oxford, UK; Maxwell House: New York, NY, USA, 1971. 
21. Bolochov, S.V.; Bronnikov, K.A. On Nonlinear Multidimensional Gravity and the Casimir Effect. Gravit. Cosmol. 2016, 22, 323-328. [CrossRef]

22. Mostepanenko, V.M.; Trunov, N.N. Casimir Effect and Its Applications; Oxford University Press: Oxford, UK, 1997 ; pp. $191-193$.

23. Chodos, A.; Detweiler, S. Where has the fifth dimension gone? Phys. Rev. 1980, 21, 2167. [CrossRef]

24. Aliyev, B.G. Where has the magnetic monopole gone? In Abstracts of the ICGAC-12; PFUR: Moscow, Russia, $2015 ;$ p. 110. 